\title{
MECANISMOS NEURALES DE CONTROL DE LA INGESTA ENERGÉTICA: ASPECTOS RELACIONADOS CON LA VÍA HOMEOSTÁTICA, HEDÓNICA Y NEUROESTEROIDOGÉNICA
}

Gisela Paola Lazzarino

gplazzarino@fbcb.unl.edu.ar

${ }^{1}$ Doctorado en Ciencias Biológicas

Director: Dr. Jorge Guillermo Ramos

Co-Director: Dra. María Florencia Andreoli

Lugar de realización: Instituto de Salud y Ambiente del Litoral / Cátedra de Bioquímica Clínica y Cuantitativa, Facultad de Bioquímica y Ciencias Biológicas, Universidad Nacional del Litoral (FBCB-UNL)

Fecha de la defensa: 27 de marzo de 2019

\section{RESUMEN}

La obesidad es la mayor amenaza para la salud pública en el mundo desarrollado. La dieta de cafetería (CAF) es un modelo dietario experimental para roedores que refleja la variedad de alimentos de alta palatabilidad y densidad energética prevalentes en la sociedad occidental. A nivel cerebral, dos sistemas regulan principalmente la ingesta: homeostático - relacionado al control hipotalámico, que regula el balance de energía incitando a comer tras el agotamiento de las reservas - y hedónico - que involucra al circuito de recompensa y puede anular a la vía homeostática durante los períodos de abundancia de energía. Los esteroides también pueden afectar la ingesta. Aunque varias enzimas neurosteroidogénicas se expresan en el hipotálamo, la relación entre sus niveles y el estado metabólico de los animales resulta desconocida. El objetivo de este trabajo de tesis fue evaluar cómo una dieta CAF administrada a corto, mediano y largo plazo afecta a los mecanismos cerebrales homeostáticos y hedónicos que regulan la ingesta de alimentos, al igual que su influencia sobre la neurosteroidogénesis hipotalámica. Además, analizamos si estos cambios podrían ser transmisibles transgeneracionalmente por mecanismos epigenéticos. Para esto, ratas Wistar hembra fueron alimentadas con alimento balanceado o CAF desde el destete y durante 4 (corto plazo), 11 (mediano plazo) o 20 (largo plazo) semanas. Los animales fueron sometidos a tests de saciedad sensorial específica, de locomoción y de interacción social. Luego de 14 semanas, hembras de cada grupo dietario fueron apareadas con machos de fertilidad comprobada y mantenidas en sus dietas durante el embarazo y la lactancia hasta el día embrionario 21 (E21) o postnatal 10 (DPN10), momento en el que se sacrificaron las crías hembra. Al finalizar la intervención dietaria los animales fueron eutanizados y sus cerebros extraídos. Por la técnica de micropunción se aislaron núcleos cerebrales discretos en los que se analizaron los niveles de ARNm de neuropéptidos hipotalámicos relacionados con la ingesta, de genes claves de la vía de recompensa cerebral, de enzimas neurosteroi- 
dogénicas y de receptores hormonales mediante PCR cuantitativa en tiempo real (qPCR). Estudios epigenéticos fueron realizados mediante digestión con enzimas de restricción sensibles a la metilación seguida de qPCR. Las hormonas séricas fueron dosadas mediante radioinmunoensayo.

A corto plazo, la dieta CAF incrementó la ingesta energética y la adiposidad, sin afectar el peso corporal. Esto se relacionó a cambios en los mecanismos de recompensa cerebral: en el área tegmental ventral (ATV) se incrementó la expresión del transportador activo de dopamina (DAT) y disminuyeron ambas isoformas de la enzima glutamato descarboxilasa, mientras que en el núcleo Accumbens (NAc) disminuyó la expresión del receptor de dopamina (DRD) 2 en la corteza (NAcSh) y aumentó la de DRD1 en el núcleo (NAcC). Los cambios en el ARNm de DAT se relacionaron con una disminución en el estado de metilación de su región promotora. Estos cambios podrían estar vinculados a las alteraciones observadas en la saciedad sensorial específica, el juego social, el comportamiento exploratorio y los niveles de ansiedad. A mediano plazo, las hembras CAF mantuvieron la ingesta energética alta e incrementaron aún más la adiposidad, presentando un estado de hiperleptinemia y sobrepeso. Esto se relacionó con una señal orexígena proveniente del Hipotálamo Lateral $(\mathrm{HL})$ y de los núcleos paraventricular (PVN) y ventromediano (VMN) del hipotálamo, dada principalmente por el incremento en la expresión de los neuropéptidos orexígenos: Orexinas (OX), proteína relacionada al Agouti (AgRP) y Neuropéptido Y (NPY), que fueron contrarrestados por un aumento en los niveles de proopiomelanocortina (POMC) en el núcleo arcuato (ARC) del hipotálamo. Además, la dieta CAF alteró la expresión de enzimas neurosteroidogénicas en el hipotálamo: en PVN los niveles de 5 $\alpha$-reductasa 1 disminuyeron y aumentaron los de citocromo p450Aromatasa (ARO), mientras que en VMN las enzimas $3 \beta$-hidroxiesteroide deshidrogenasa (3ß-HSD) y ARO aumentaron su expresión. Todos estos resultados se asociaron con cambios en los niveles de metilación de las regiones promotoras de los genes, indicando que mecanismos epigenéticos podrían estar involucrados. En el largo plazo, estos cambios metabólicos y moleculares se intensificaron, manifestándose un cuadro de obesidad. Además, se encontró una alteración en los niveles de AgRP, el cual duplicó su expresión en PVN mientras que disminuyó en ARC. También se encontró un aumento en

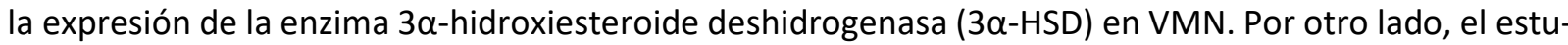
dio de la progenie demostró que la dieta materna CAF disminuyó significativamente el peso corporal de las crías y afectó al sistema homeostático, ya que la expresión de POMC se redujo en E21, cambio que se mantuvo en DPN10. En DPN10 la vía dopaminérgica también se vio afectada: en el ATV la dieta materna CAF disminuyó la transcripción de la enzima tiroxina hidroxilasa (TH), DRD2 y DAT; y en NAc, redujo la expresión de DRD1 y DRD2. Además, los cambios en la expresión de TH y DRD2 se relacionaron con modificaciones en el estado de metilación de sus regiones promotoras. Nuestros resultados muestran que la ingesta de una dieta de alta palatabilidad desde etapas tempranas de la vida altera de manera diferencial a los sistemas cerebrales involucrados en el control de la ingesta. Además, algunos de los 
cambios observados serían transmisibles transgeneracionalmente. Finalmente, resulta esencial identificar los cambios en la expresión génica al comienzo del desarrollo de la obesidad, para poder predecir la trayectoria de esta patología y así elegir una terapia eficaz.

\section{ABSTRACT}

\section{Neural mechanisms involved in the control of energy intake: aspects related to the homeo- static, hedonic and neurosteroidogenic pathways}

The aim of this thesis was to evaluate the effects of cafeteria diet (CAF) intake from weaning on the homeostatic and hedonic brain mechanisms that regulate food intake, as well as its influence on hypothalamic neurosteroidogenesis, at different feeding periods in female Wistar rats. Besides, we analyzed whether these changes could be transmissible transgenerationally. The mRNA levels and DNA methylation state of feeding-related neuropeptides, key genes of the reward pathway, neurosteroidogenic enzymes, and hormone receptors were analysed by QPCR in discrete brain nuclei isolated by micropunching technique. Our results showed that the intake of CAF from early-stages of life differentially alters the brain systems involved in food intake control depending on dietary intervention. Four weeks of CAF (short-term) increased energy intake and adiposity, deregulating key genes of the reward pathway, at least in part via epigenetic changes. Eleven weeks of CAF (medium-term) increased energy intake, adiposity, leptinemia, and body weight, deregulating the homeostatic and the neurosteroidogenic pathways. Metabolic and molecular changes were intensified after 20 weeks of diet (long-term), when obesity was already manifested. Moreover, epigenetic changes were observed in the animals fed with CAF in the medium and long-term, suggesting that an extended intake of this diet could differentially affect the DNA methylation pattern of the promoter regions in discrete hypothalamic nuclei. Furthermore, these changes were transmissible transgenerationally. The alterations in these brain nuclei could add information about their differential role in food intake control, and how their action is disrupted during the development of obesity. 